\title{
CROP TYPE MAPPING FROM A SEQUENCE OF TERRASAR-X IMAGES WITH DYNAMIC CONDITIONAL RANDOM FIELDS
}

\author{
B. K. Kenduiywo ${ }^{a *}$ D. Bargiel ${ }^{\mathrm{a}}$, U. Soergel ${ }^{\mathrm{b}}$ \\ ${ }^{a}$ Institute of Geodesy, Technische Universität Darmstadt, Germany - (kenduiywo, bargiel)@ geod.tu-darmstadt.de \\ ${ }^{\mathrm{b}}$ Institute for Photogrammetry, University of Stuttgart, Germany - soergel@ifp.uni-stuttgart.de
}

\author{
Commission VII, WG VII/4
}

KEY WORDS: Dynamic Conditional Random Fields (DCRFs), conditional random fields (CRFs), phenology, spatial-temporal classification, TerraSAR-X

\begin{abstract}
:
Crop phenology is dynamic as it changes with times of the year. Such biophysical processes also look spectrally different to remote sensing satellites. Some crops may depict similar spectral properties if their phenology coincide, but differ later when their phenology diverge. Thus, conventional approaches that select only images from phenological stages where crops are distinguishable for classification, have low discrimination. In contrast, stacking images within a cropping season limits discrimination to a single feature space that can suffer from overlapping classes. Since crop backscatter varies with time, it can aid discrimination. Therefore, our main objective is to develop a crop sequence classification method using multitemporal TerraSAR-X images. We adopt first order markov assumption in undirected temporal graph sequence. This property is exploited to implement Dynamic Conditional Random Fields (DCRFs). Our DCRFs model has a repeated structure of temporally connected Conditional Random Fields (CRFs). Each node in the sequence is connected to its predecessor via conditional probability matrix. The matrix is computed using posterior class probabilities from association potential. This way, there is a mutual temporal exchange of phenological information observed in TerraSAR-X images. When compared to independent epoch classification, the designed DCRF model improved crop discrimination at each epoch in the sequence. However, government, insurers, agricultural market traders and other stakeholders are interested in the quantity of a certain crop in a season. Therefore, we further develop a DCRF ensemble classifier. The ensemble produces an optimal crop map by maximizing over posterior class probabilities selected from the sequence based on maximum F1-score and weighted by correctness. Our ensemble technique is compared to standard approach of stacking all images as bands for classification using Maximum Likelihood Classifier (MLC) and standard CRFs. It outperforms MLC and CRFs by $7.70 \%$ and $6.42 \%$ in overall accuracy, respectively.
\end{abstract}

\section{INTRODUCTION}

Food security is a matter of concern globally. Shortage of food can lead to socio-economic consequences. Foresight by the world bank estimates that since 2010 demand for food has increased resulting into extreme poverty of about 44 million people (World-Bank, 2011). Estimated rise in population and diets will require significant increase in food production (Tilman et al., 2011). Therefore, current efforts by farmers, agronomist and related stakeholders is to ensure that food production is optimal and sustainable. This demands regular update of spatial and temporal information on agriculture activities to aid monitoring and sustainable food policy decision making. Such information is to be derived from a dynamic phenomenon over a vast area. Thus, methods of data collection that can match this scale are necessary.

Remote sensing satellites capture spectral, spatial and temporal attributes of phenomena on the earth surface. This is because of their specific electromagnetic spectrum sensitivity, synoptic view and temporal capability. Mapping agriculture activities requires insights on crop dynamics, e.g. phenology ${ }^{1}$ states and seasonal growth. Spectral trend of agricultural parcels is constantly changing. Different crops may at a given time be in the same phenological state, depicting similar spectral attributes, but differ significantly in another time (Siachalou et al., 2015). According to Siachalou et al. (2015), crop mapping using single-date remote sensing images, even if acquired in critical growth stages, can not

\footnotetext{
* Corresponding author.

${ }^{1}$ In remote sensing we can consider phenology as the appearance of a crop at a particular instance in its life cycle.
}

offer optimal results in case of crops with similar phenology. A sequence of multitemporal images and phenological information can be integrated using a robust statistical framework to improve crop discrimination.

This study adopts a sequence of Synthetic Aperture Radar (SAR) images from TerraSAR-X satellite for crop classification. Radar satellites are daylight and weather independent. Signals from radar can penetrate vegetation canopy and dry soil thus bearing volumetric and subsurface information. These attributes renders SAR a good medium to deliver a sequence of images of highest temporal density suitable for crop classification regardless of climatic zones. In contrast, complexity, e.g. speckle interference, accompanying SAR data challenges conventional pixel based approaches. Spatial context can be employed to overcome it (Kenduiywo et al., 2014). However, crops in the same phenology exhibit correlated SAR backscatter. Conventional approaches stack multitemporal images as bands for classification (e.g., Bargiel and Herrmann (2011); Forkuor et al. (2014); Sonobe et al. (2014)). By doing so, significant temporal information from satellite observed crop phenology is limited to a single feature space. Discrimination in such feature space can suffer from overlapping class boundaries due to large class variance from spectral variation. In addition to spatial context, integration of temporal context in a principled manner can help resolve classes over time.

Studies like Leite et al. (2011); Siachalou et al. (2015) incorporate crop phenology via Hidden Markov Models (HMM). They used HMM to model temporal context among crop phenology stages but lack a proper spatial context framework. Mono-temporal crop 
classification adopting spatial context also exist (Ozdarici-Ok et al., 2015; Roscher et al., 2010). Efforts to use both spatial and temporal context to classify crops from optical images is done in (Hoberg and Müller, 2011). The study models temporal context via a global transition matrix determined from training data. A global matrix assumes similar phenology transitions over all pixels neglecting changes that may exist in the image. In our previous study (Kenduiywo et al., 2015), pixel-wise temporal information exchange between grouped crop classes in two epochs ${ }^{2}$ are considered. Here, the approach is extended to a dynamic template that classifies each crop type over a sequence of multitemporal SAR images.

The contributions of this study are threefold: (1) to develop a spatial-temporal classification framework to discriminate crops using a sequence of multitemporal TerraSAR-X images, (2) design a suitable spatial interaction model, modified from contrast sensitive model suggested by Shotton et al. (2009), to moderate changes in class labels based on data evidence and (3) to design an ensemble framework to generate an optimal season crop map. We used dynamic conditional random field(s) (DCRFs) for sequence crop classification. The framework allows reasoning via Bayesian theory in a principled statistical manner under uncertainty. We incorporate DCRF into standard conditional random fields (CRFs) as a temporal classifier template. This forms a robust spatial-temporal sequence crop classifier termed as DCRF because: (a) of a changing probabilistic relational model between nodes in the sequence, (b) the model captures time-changing phenomena, encodes complex interactions over the set of all possible classes and data and uncertainty in a principled manner, and (c) the model is a conditional distribution that factorizes according to an undirected graphical model whose structure and parameters are repeated over a sequence (Sutton et al., 2007).

The rest of the paper is organized as follows. Section 2 introduces the approach we adopted and illustrates how CRF is designed for spatial-temporal crop sequence classification. Section 3 describes selected study area, crops considered for classification, data and features used, details of experiments conducted by our design and other methods. In Section 4, a description of results from our technique compared to others is made leading to a discussion in Section 5. Conclusions from the study and future tasks are provided in Section 6.

\section{METHODS}

\subsection{Conditional Random Fields}

Conditional Random Fields were introduced by Lafferty et al. (2001) for one-dimensional text classification and extended to two-dimensional image classification (Kumar, 2006). They are undirected graphs which represent conditional probability distribution over a set of data/data sequence. The conditional probabilities are represented in non-negative functions, potentials, defined over a subset of fully connected variables known as cliques ${ }^{3}$. In CRFs, posterior probability of a distribution is computed as a product of potentials through inference techniques.

Definition Consider a sequence of images $\mathbf{x}=\mathbf{x}_{t}$ modelled over corresponding discrete labels $\mathbf{y}=\mathbf{y}_{t}$, from a given set of class labels $l \in m$, acquired at different times $t$ where $t=1, \ldots, T$. Let $G=\{S, E\}$ be a graph with spatial edges $E$ defined over a pair of cliques $i$ and $j$ in a neighbourhood set $N$ such that $\mathbf{y}=$ $\left(y_{i}\right)_{i \in S}$ where $\mathbf{y}$ is indexed by nodes (vertices) $S$ of the Graph

\footnotetext{
${ }^{2} \mathrm{An}$ epoch is an image date within a sequence of acquired images.

${ }^{3} \mathrm{~A}$ clique is a fully connected subgraph.
}

$G$. In mono-temporal classification, the random variable $(\mathbf{y}, \mathbf{x})$ is a CRF only if, when conditioned on $\mathbf{x}$, the random variable $y_{i}$ obeys the Markov property with respect to $\mathrm{G}: P\left(y_{i} \mid \mathbf{x}, \mathbf{y}_{\backslash i}\right)=$ $P\left(y_{i} \mid \mathbf{x}, \mathbf{y}_{N_{i}}\right)$, where $\mathbf{y}_{\backslash i}$ is the set of all nodes in the $\mathrm{G}$ except node $i$ and $N_{i}$ is a set of neighbours of node $i$ in G.

Following Hammersley and Clifford basic theorem, the joint distribution over the labels $\mathbf{y}$ given the data $\mathbf{x}$ can be written as:

$$
P(\mathbf{y} \mid \mathbf{x})=\frac{1}{Z(\mathbf{x})} \exp \left\{\sum_{i \in S} A\left(y_{i}, \mathbf{x}\right)+\sum_{i \in S} \sum_{j \in N_{i}} I\left(y_{i}, y_{j}, \mathbf{x}\right)\right\}
$$

where $Z(\mathbf{x})$ is a normalizing constant referred to as partition function, and $A$ and $I$ are the association (unary) and interaction (pairwise) potentials respectively.

\subsection{Dynamic conditional random fields}

Sequence classification requires determination of posterior probability $P\left(\mathbf{y}_{1, \ldots, T} \mid \mathbf{x}_{1, \ldots, T}\right)$. The computation is intractable and exponential in time as it involves estimation of $S^{T}$ functions in a 2D space. Since satellite observation of crops is unique in each instance, we assume that their evolution is independent. In this way, the conventional class conditional independence (Swain, 1978) can be attained: $P\left(\mathbf{y}_{1, \ldots, T} \mid \mathbf{x}_{1, \ldots, T}\right)=P\left(\mathbf{y}_{1} \mid \mathbf{x}_{1}\right), \ldots, P\left(\mathbf{y}_{T} \mid \mathbf{x}_{T}\right)$. This simplifies the classification problem to an independent estimation of class posterior probabilities $P\left(y_{i}\right)$ for each node $i$ in $t$. Spatial interactions are also considered at each epoch by $I$ in Eq. (1).

To exploit crop phenological information, we extend DCRFs proposed by Sutton et al. (2007) for text sequence classification to 3-D image sequence classification. A DCRF is a conditionallytrained undirected graphical model whose structure and parameters are repeated over a sequence. We developed an undirected DCRF graph template that factorizes according to first order Markov assumption. In the design, each node $i$ at time $t$ can depend on node data from the previous (if $t \neq 0$ ) and subsequent (if $t \neq T$ ) epochs. The objective is to connect a set of all possible temporal cliques $C$ of nodes $S$ using a conditional probability matrix distribution $P\left(\mathbf{y} \mid \mathbf{y}_{t-1}, \mathbf{x}, \mathbf{x}_{t-1}\right)$. This set-up gives a DCRF sequence template model, considering crop phenology, such that a node can have at least one or two temporal neighbours (Fig. 1).

Definition Let $c, c \in C$, be a temporal clique index of a node $k$ in epoch $\Delta t=t-1$ of a label vector $\mathbf{y}_{\Delta t}$ which corresponds to another node $i$ in label vector $\mathbf{y}_{t}$ at time $t$ such that $c=\{k, \Delta t\}$. In this case, a set of random variables $\mathbf{y}_{i, t, c} \equiv\left\{y_{i, t} \mid(k, \Delta t) \in c\right\}$ is the set of variables of the evolving clique index $c$ at time $t$ in the sequence $T$. Then, our spatial-temporal DCRF template can be expressed as:

$$
\begin{array}{r}
P(\mathbf{y} \mid \mathbf{x})=\frac{1}{Z(\mathbf{x})} \exp \left\{\sum_{i \in S} A\left(y_{i}, \mathbf{x}\right)+\sum_{i \in S} \sum_{j \in N_{i}}\right. \\
\left.I\left(y_{i}, y_{j}, \mathbf{x}\right)+\sum_{t \in T} \sum_{i \in S} \sum_{c \in C} T P\left(y_{i, t, c}, \mathbf{x}, \mathbf{x}_{\Delta t}\right)\right\}
\end{array}
$$

where $T P$ is temporal potential.

2.2.1 Association Potential determines how likely an image site $i$ takes a label $y_{i}$ given the data $\mathbf{x}: A\left(y_{i}, \mathbf{x}\right)=P\left(y_{i} \mid \mathbf{f}_{i}(\mathbf{x})\right)$, $\mathbf{f}_{i}(\mathbf{x})$ is a site-wise feature vector (Kumar, 2006). We used random forest (RF) (Breiman, 2001) to determine $A$ by independent classification of different epochs assuming that evolution of crops 


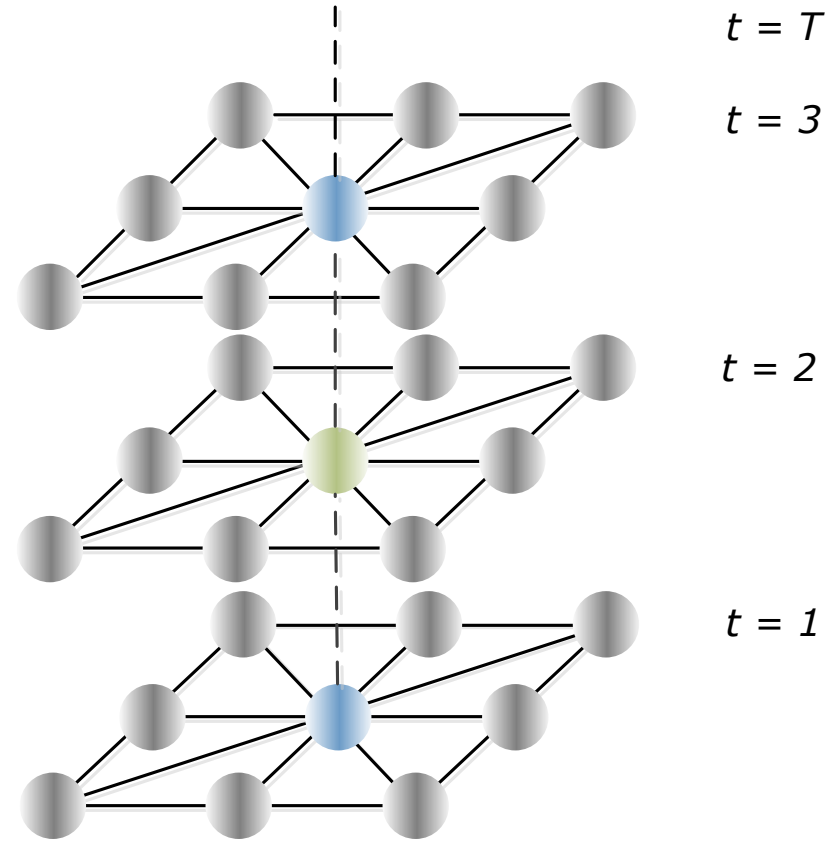

Figure 1. First order temporal neighbours of a node at different times $t$ of a sequence $T$. Spatial and temporal edges are indicated by solid and dashed lines respectively.

are unique over the sequence. A RF conducts classification by casting votes from a number of decision trees $D_{T}$ generated during training. If the number of votes cast for a given class label $y$ by RF is $V_{y}$, then our $A$ at site $i$ is $P\left(y_{i}=y \mid \mathbf{f}_{i}(\mathbf{x})\right)=\left(\frac{V_{y}}{D_{T}}\right)$. We set $D_{T}=250$ because over 200 trees RF stabilizes (Hastie et al., 2011) and set tree depth as 25 .

2.2.2 Interaction Potential measures the influence of data and neighbouring labels on site $i$. It ensures that site $i$, as initially determined by association potential, is labelled to its corresponding "true class" given data evidence $\mathbf{x}$ and neighbourhood dependency $N$ where $j \in N_{i}$. We set $N=8$, second order neighbourhood structure, as shown in Fig. 1. This study modelled $I$ by comparing two models: contrast sensitive model suggested in (Shotton et al., 2009),

$$
I\left(y_{i}, y_{j}, \mathbf{x}\right)= \begin{cases}\beta \cdot \exp \left(-\eta \cdot d_{i j}\right) & \text { if } y_{i}=y_{j} \\ 0 & \text { if } y_{i} \neq y_{j}\end{cases}
$$

and a new version of contrast sensitive Potts model:

$I\left(y_{i}, y_{j}, \mathbf{x}\right)= \begin{cases}\beta \cdot \exp \left(-\eta \cdot d_{i j}\right) & \text { if } y_{i}=y_{j} \\ \beta \cdot\left(\operatorname{Max}\left[1-\exp \left(-\eta \cdot d_{i j}\right), \epsilon\right]\right)^{-1} & \text { if } y_{i} \neq y_{j}\end{cases}$

where $\beta$ is a spatial interaction parameter that regulates smoothness, parameter $\eta$ weights and controls inclusion $(\eta>0)$ or exclusion $(\eta=0)$ of data interactions $d_{i j}=\frac{\sqrt{\sum_{i=1}^{\mathbf{R}}\left|\mathbf{f}_{i}(\mathbf{x})-\mathbf{f}_{j}(\mathbf{x})\right|^{2}}}{\mathbf{R}}$ of adjacent node features $\mathbf{f}_{i}$ and $\mathbf{f}_{j}, \mathbf{R}$ is the number of features/elements in vectors $\mathbf{f}_{i}$ and $\mathbf{f}_{j}, \operatorname{Max}$ is a function that returns a maximum between two values and $\epsilon$ is a value close to zero (it prevents division by zero). Since data interactions are considered in CRF, by default $\eta>0$. Division by $\mathbf{R}$ ensures identical influence of $I$ over the sequence of images $T$. Therefore, the model is different from contrast sensitive Potts model because transitions of adjacent labels are now moderated based on data evidence both when initial labels from $A$ are similar or dissimilar. In this man- ner, the model regulates smoothing while preserving edges.

2.2.3 Temporal Potential models interactions between nodes in the sequence of images $T$. This potential can be considered as a classifier that ensures mutual information exchange between nodes $i$ and $k$ in epoch $t$ and $\Delta t$. Since the posterior probabilities $P\left(y_{i}=l\right)$ and $P\left(y_{k}=l\right)$ are determined by $A$, our $T P$ can be expressed as:

$$
T P\left(y_{i, t, c}, \mathbf{x}, \mathbf{x}_{\Delta t}\right)=P\left(y_{i}=l \mid y_{k}=l\right)
$$

To solve Eq. (5), Bayesian formula is used to compute conditional probability matrix of $T P$ as:

$$
P\left(y_{i}=l \mid y_{k}=l\right)=\frac{P\left(y_{i}=l, y_{k}=l\right)}{\sum_{y_{i}} P\left(y_{i}=l, y_{k}=l\right)}
$$

Eq. (6) determines the probability of a crop label $l \in m$ being assigned to a node $i$ given data from the two epochs $t$ and $\Delta t$. It is used to compute a pixel-wise conditional probability matrix as shown in (Kenduiywo et al., 2015).

\subsection{Optimal crop mapping with DCRF}

In most cases farmers, governments, and other stakeholders are interested in the quantity of a certain crop in a given season. Our DCRF approach incorporates phenological information exchange between each epoch given a preceding one and vice versa. At each epoch we obtain posterior class probabilities incorporating phenological information in images. For this reason, we develop an ensemble classifier to generate an optimal seasonal crop map.

Consider our classification problem where each node $i \in S$ is to be assigned a discrete class label $y$ from $l \in m$ possible classes $\left(y_{1}, \ldots, y_{m}\right)$ in each epoch $t$ from the sequence $T$. Now given that posterior probabilities $P\left(y_{l} \mid x, t\right)$ have been determined, then for each class $l$ we select a probability with maximum F1-score from the sequence $T$ and weight them with correctness accuracy. Then, a discrete class for node $i$ can be determined by maximizing over probabilities selected from the sequence. Since the same training sites are used throughout the sequence (crop season), then prior probability are assumed equal:

$$
\hat{y}_{i}=\underset{l=1}{\arg \max _{\max }}\left\{\max _{t=1}^{T} F 1\left[P\left(y_{l} \mid \mathbf{x}\right), t\right] * \text { Corr }\right\}
$$

where $F 1\left[P\left(y_{l} \mid \mathbf{x}\right), t\right]$ is a probability corresponding to class $l$ with maximum F1-score (Sokolova et al., 2006), i.e. $F 1=$ $\frac{2 \text { (correctness } \times \text { completeness })}{\text { correctness+completeness }}$, at time $t$, Corr is the correctness accuracy measure and $\hat{y}_{i}$ the estimated class label. Correctness and completeness correspond to recall and precision respectively. This approach, Eq. (7), is compared to the following classifier combinations rules in (Kittler et al., 1998):

$$
\begin{aligned}
& \text { Max rule: } \hat{y}_{i}=\underset{l=1}{\arg \max }\left\{\max _{t=1}^{T} P\left(y_{l} \mid \mathbf{x}, t\right)\right\} \\
& \text { Majority vote: } \hat{y}_{i}=\underset{l=1}{\arg \max _{\max }^{m}}\left\{\sum_{t=1}^{T} \Delta_{l t}\right\} \\
& \text { Median rule: } \hat{y}_{i}=\underset{l=1}{\arg \max }\left\{\operatorname{med}_{t=1}^{T} P\left(y_{l} \mid \mathbf{x}, t\right)\right\} \\
& \text { Product rule: } \hat{y}_{i}=\underset{l=1}{\arg \max }\left\{\prod_{t=1}^{T} P\left(y_{l} \mid \mathbf{x}, t\right)\right\}
\end{aligned}
$$

\subsection{Training and Inference}

Solution to Eq. (2) is obtained by maximizing probabilities, spectral $(A)$, spatial $(I)$ and temporal $(T P)$ using Bayes' Maximum 
A Posterior (MAP) estimate. This requires an inference algorithm to determine posterior probabilities $P(\mathbf{y} \mid \mathbf{x})$ and a maximization algorithm to estimate optimum labels $\hat{y}$. We apply sum-product Loopy Belief Propagation (LBP) (Murphy et al., 1999), a standard inference algorithm in graphs with cycles. To estimate class labels, we design a maximization algorithm. The association potential probabilities used in both $I$ and $T P$ are trained using RF implemented in OpenCV (OpenCV, 2014).

\section{EXPERIMENTS}

\subsection{Study area and data}

The study area is located in Northern Germany $\left(52.26^{\circ} \mathrm{N}\right.$, $9.84^{\circ} \mathrm{E}$ ), see Fig. 2. The average annual precipitation and temperature are $656 \mathrm{~mm}$ and $8.9^{\circ} \mathrm{C}$ respectively (Deutscher Wetterdienst, 2012). The region is characterized by intensive agriculture with large farms. Crops in the area include: 1) barley, 2) canola, 3) grassland, 4) maize, 5) oat, 6) potato, 7) rye 8) sugar beet and, 9) wheat. These crops go through different phenological stages within a season, a fact that can enhance discrimination. Four phenology phases, preparation, seeding, growing, harvesting and post harvest, are considered (Fig. 3). Preparation phase involves ploughing and soil grooming processes before seeding. In seeding phase, crop seeds are placed in the soil. Growing phase includes the period between crop germination to ripening. After ripening, harvesting starts by gathering mature crops from the fields. The last stage is post harvest phase, where the field could be fallow or with some remaining ripe crops.
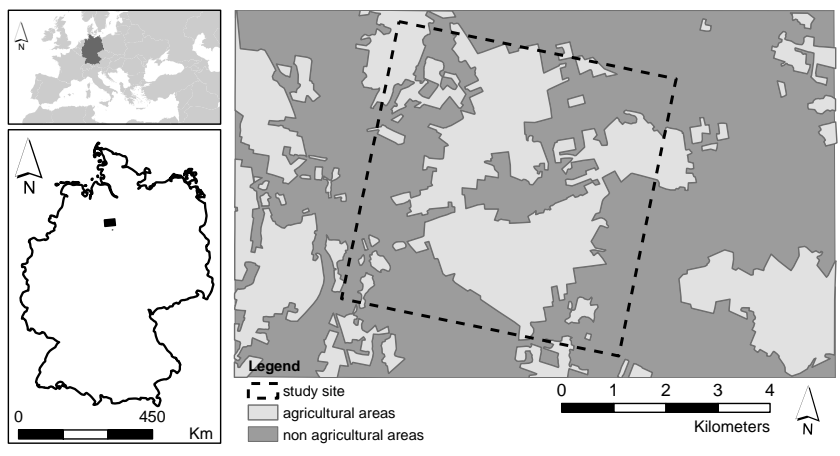

Figure 2. Study area.

\begin{tabular}{|c|c|c|c|c|c|c|c|c|c|c|c|c|}
\hline & Jan & Feb & Mar & Apr & May & Jun & Jul & Aug & Sep & Oct & Nov & Dec \\
\hline \multicolumn{13}{|l|}{ Maize } \\
\hline \multicolumn{13}{|l|}{ Potato } \\
\hline \multicolumn{13}{|l|}{ Canola } \\
\hline \multicolumn{13}{|l|}{ Sugar beet } \\
\hline \multicolumn{13}{|l|}{ Oat } \\
\hline \multicolumn{13}{|l|}{ Barley } \\
\hline \multicolumn{13}{|l|}{ Wheat } \\
\hline \multicolumn{13}{|l|}{ Rye } \\
\hline \multicolumn{13}{|l|}{ Grassland } \\
\hline & Jan & Feb & Mar & Apr & May & Jun & Jul & Aug & Sep & Oct & Nov & Dec \\
\hline
\end{tabular}

Figure 3. Phenology stages of crops considered for classification.

The temporal sequence consists of six dual polarized ( $\mathrm{HH}$ and VV) TerraSAR-X High Resolution Spotlight images acquired on: $11^{t h}$ March, $13^{t h}$ April, $22^{\text {nd }}$ May, $18^{t h}$ June, $10^{\text {th }}$ June and $17^{\text {th }}$ October in the year 2009. All are acquired at an incidence of $34.75^{\circ}$ with range and azimuth resolutions of $2.1 \mathrm{~m}$ and 2.4 $\mathrm{m}$ except for the month of May which has an incidence angle of $43.65^{\circ}$ with range and azimuth resolutions of $3.4 \mathrm{~m}$ and $2.9 \mathrm{~m}$. The images were delivered as ground range products (MGD) with equidistant pixel spacing. They are radiometrically calibrated to $\sigma^{0}$ according to Fritz and Eineder (2009). All images are coregistered to an extent of $7.1 \times 11.8 \mathrm{~km}^{2}$ using WGS $1984 \mathrm{da}-$ tum on UTM Zone $32 \mathrm{~N}$ coordinate projection system. Our experiment site covers an extent of $5.4 \times 5.4 \mathrm{~km}^{2}$.

Reference data campaign was conducted concurrently with image acquisition during the year. The reference parcels were separated into training and validation sets using stratified random sampling design tool in ArcGIS 10.0 (Buja and Menza, 2013). Distribution of each crop type (training set / validation set) in hectares is: barley (38.54 / 41.30), canola (38.60 / 40.87), grassland (69.97 / $55.10)$, maize $(27.63$ / 33.10), oat (10.11 / 17.39), potato (55.76/ $66.45)$, rye ( 97.23 / 79.04), sugar beet (52.49 / 47.51), and wheat (34.88 / 34.84).

\subsection{Feature selection}

Gray Level Co-occurence Measures (GLCM) were computed using a $3 \times 3$ matrix. Eight features - mean, variance, correlation, homogeneity, contrast, dissimilarity, entropy and 2nd moment were computed in directions $0^{\circ}, 45^{\circ}, 90^{\circ}$, and $135^{\circ}$ giving rise to a total of 32 features in each polarization. Random Forest variance importance was used to select 4 significant features from the 8 GLCM features in each direction and polarization (a total of 32 features per epoch). Important GLCM features as per RF include: correlation, homogeneity, variance and mean. For each selected feature, a super pixel/block was generated from a mean of $3 \times 3$ pixels. Block size selection was done in consideration to the minimum mappable unit. The shift from pixels to block segments classification is advocated in (Blaschke and Strobl, 2001).

\subsection{Parameter determination}

Selection of a model for $I$ and its parameters is an important step in CRF classification. Thus, we conducted classification tests using models in Eqs. (2) and (3) over a range of $\beta$ and $\eta$ parameter values in 2-D logarithmic scale. Epochs in growing season (June and July) are adopted to compute average overall classification accuracy for each set of parameters. These epochs were chosen because within the period, returned radar backscatter are dominantly from crops. A suitable model of $I$ including $\beta$ and $\eta$ parameters are then selected using initial 2-D logarithmic scale search results.

\subsection{Classification}

We adopt the technique in Eq. (2) for crop sequence classification. The approach classifies each epoch integrating first order temporal information and spatial information from 8 neighbouring nodes. We compare this approach to MLC, RF (association potential) and mono-temporal CRF (CRF-mono), Eq. (1), in single epoch classification.

Posterior probabilities from each epoch were combined to generate an optimal map. Different methods introduced in Section 2.3 were tested and compared to our new approach in Eq. (7). Moreover, studies like Bargiel and Herrmann (2011); Forkuor et al. (2014); Sonobe et al. (2014) stacked temporal images/features as bands for multitemporal classification. Therefore, we also stacked a sequence of amplitude images for classification using MLC (MLC-stack) and CRF (CRF-stack). This technique is compared to optimal sequence classification method introduced in Eq. (7). We term the approach DCRF max F1-score. 


\section{RESULTS}

\subsection{Feature selection results}

Results of GLCM features selection are described here. Four features were selected using RF variable importance criteria. Fig. 4 shows RF importance computed from an average of four directions, $0^{\circ}, 45^{\circ}, 90^{\circ}$, and $135^{\circ}$, for each feature and subsequently their average over epochs. Generally with the exception of $\mathrm{HH}$-polarized correlation features, features computed from VV-polarization backscatter have a higher importance compared to features computed from HH-polarization.

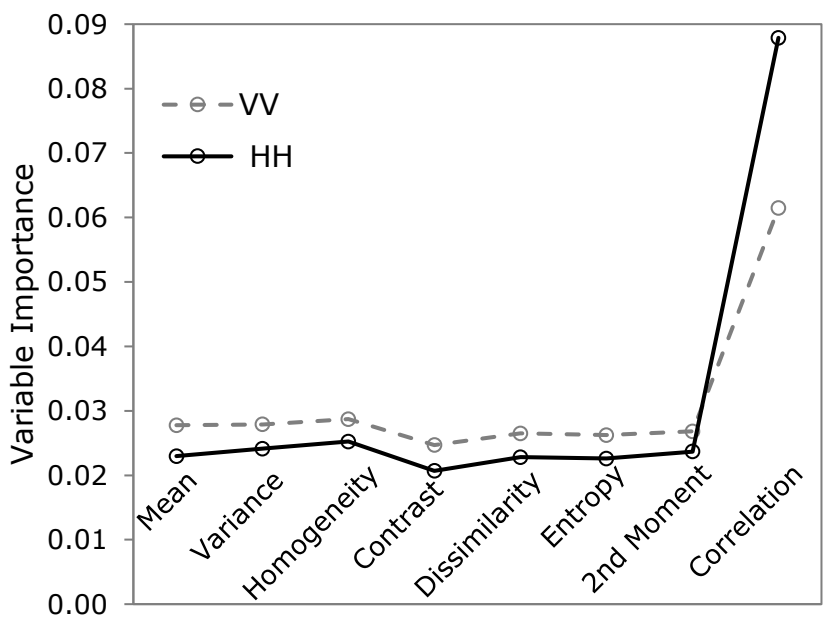

Figure 4. Average random forest variable importance for different GLCM features.

\subsection{Parameter determination results}

Selection of a data interaction model for $I$ and its corresponding parameters was guided by results in Fig. 5. The new expanded contrast sensitive model, Eq. (4), outperforms standard contrast sensitive, Eq. (3), in overall accuracy (OA) with most parameters. Our new model gives high classification accuracy for $10^{1} \leq$ beta $\leq 10^{3}$ and $10^{-2} \leq$ eta $\leq 5$. In contrast, accuracy for contrast sensitive Potts model reduces if beta $>0.1$ in combination with any eta parameter values. This search, with a compromise between high accuracy and over-smoothing, guided our choice of $\beta=10$ and et $a=1$ for our new data dependent interaction model in Eq. (4). We use these parameter values across the sequence for comparability.

\subsection{Classification results}

Results from DCRF epoch classification compared to other approaches are illustrated in Fig. 6. The results show that DCRF approach outperforms CRF-mono, RF and MLC. In all epochs MLC has the least accuracy followed by RF and CRF-mono respectively. The addition of temporal information also improved classification accuracy in each epoch since DCRF outperformed CRF-mono which considers only spatial information. Spatial information also improved classification as demonstrated by CRFmono performance compared to RF and MLC which have lower accuracy.

An optimal crop map is generated from epoch-wise DCRF posterior probabilities using different classifier combination strategies as depicted by results in Table 1 . The technique we introduce, DCRF max F1-score, outperforms max rule, majority vote, product rule and median rule by $33.92 \%, 10.04 \%, 7.91 \%$ and $7.85 \%$ in

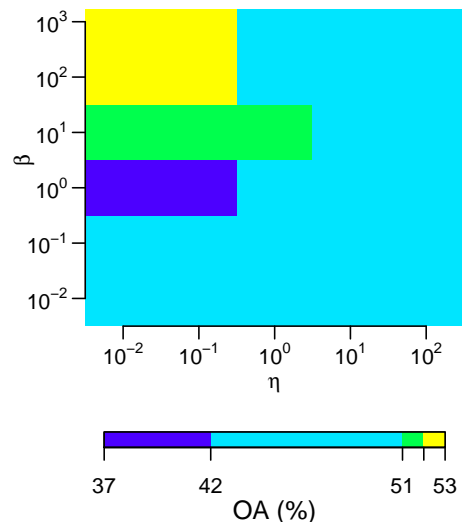

(a)

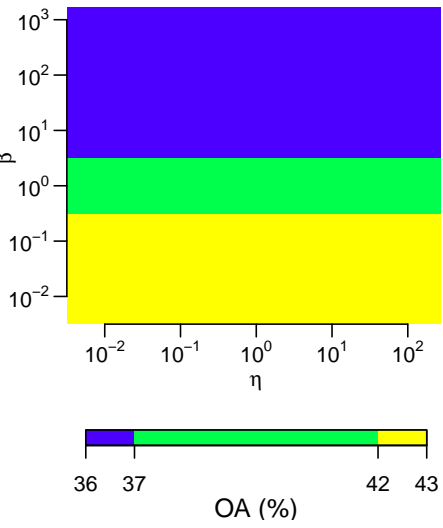

(b)
Figure 5. Comparison of our new spatial interaction model Fig. 5a and contrast Potts model Fig. 5b over a logarithmic scale.

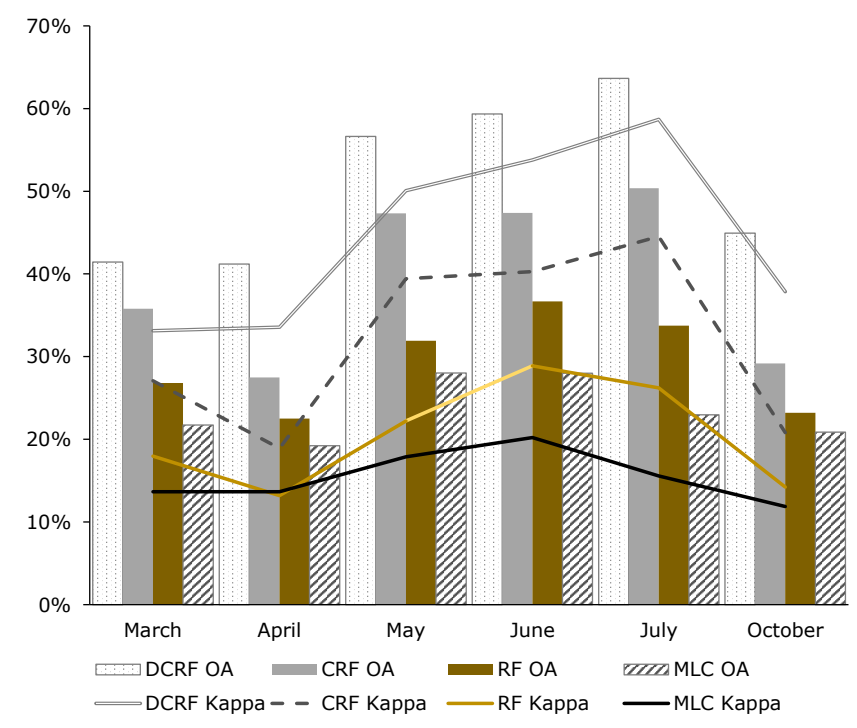

Figure 6. Epoch-wise classification results, overall accuracy and kappa, from different approaches.

overall accuracy respectively. Thus, max rule has the least overall accuracy followed by majority vote, median rule and product rule respectively.

\begin{tabular}{lll}
\hline Method & OA & Kappa \\
\hline Max Rule & $40.61 \%$ & $33.59 \%$ \\
Majority Vote & $64.49 \%$ & $59.41 \%$ \\
Median Rule & $66.68 \%$ & $61.80 \%$ \\
Product Rule & $66.62 \%$ & $61.71 \%$ \\
Max F1-Score & $74.53 \%$ & $70.98 \%$ \\
\hline
\end{tabular}

Table 1. Comparison of different strategies of integrating DCRF posterior probabilities to produce an optimal classification map.

Designed DCRF max F1-score classifier ensemble is compared to the conventional approach of stacking multitemporal images as input bands to a classifier. Table 2 illustrates that DCRF max F1score outperforms MLC and CRF methods adopting stacked images by overall accuracy of $7.70 \%$ and $6.42 \%$ respectively. Further comparison of DCRF max F1-score and MLC-stack using correctness and completeness of each class is made in Table 3. This exposes in detail how each crop is recognized by the clas- 
sifiers as opposed to a limited view on overall accuracy in Table 2 . It is only grassland that has a lower classification accuracy in our approach (correctness $-16.4 \%$ / completeness $-2.3 \%$ ) compared to MLC-stack approach. All other classes were classified better or comparable to MLC-stack approach. This is especially true for barley $(+17.05 \% /+2.01 \%)$, maize $(+24.33 \% /+18.58 \%)$, oat $(+19.99 \% /+13.45 \%)$ and sugar beet $(+45.83 \% /+21.93 \%)$. Rye and wheat also slightly improved $(+5.58 \% /+2.03 \%)$ and $(+5.65 \% /+5.80 \%)$ respectively, while canola and potato exhibit a lower correctness of $-5.88 \%$ and $-3.40 \%$ but a higher completeness of $+2.70 \%$ and $+10.70 \%$.

\begin{tabular}{lll}
\hline Method & OA & Kappa \\
\hline MLC-stack & $66.83 \%$ & $61.67 \%$ \\
CRF-stack & $68.11 \%$ & $63.33 \%$ \\
DCRF max F1-Score & $74.53 \%$ & $70.98 \%$ \\
\hline
\end{tabular}

Table 2. Comparison of DCRF max F1-score to stacking multitemporal images together as input bands for classification.

\begin{tabular}{lcccc}
\hline & \multicolumn{2}{c}{ Correctness } & \multicolumn{2}{c}{ Completeness } \\
\hline Class & $\begin{array}{c}\text { DCRF } \\
\text { max F1-score }\end{array}$ & $\begin{array}{c}\text { MLC } \\
\text { stack }\end{array}$ & $\begin{array}{c}\text { DCRF } \\
\text { max F1-score }\end{array}$ & $\begin{array}{c}\text { MLC } \\
\text { stack }\end{array}$ \\
\hline Barley & $73.49 \%$ & $56.44 \%$ & $70.61 \%$ & $68.60 \%$ \\
Canola & $91.11 \%$ & $96.99 \%$ & $97.78 \%$ & $95.10 \%$ \\
Grassland & $77.88 \%$ & $94.34 \%$ & $86.49 \%$ & $88.79 \%$ \\
Maize & $47.55 \%$ & $23.22 \%$ & $50.32 \%$ & $31.74 \%$ \\
Oat & $61.11 \%$ & $41.12 \%$ & $89.91 \%$ & $76.46 \%$ \\
Potato & $74.78 \%$ & $78.18 \%$ & $63.45 \%$ & $52.80 \%$ \\
Rye & $75.24 \%$ & $69.66 \%$ & $65.05 \%$ & $63.02 \%$ \\
Sugar beet & $87.09 \%$ & $41.26 \%$ & $89.87 \%$ & $67.94 \%$ \\
Wheat & $66.93 \%$ & $61.28 \%$ & $70.13 \%$ & $64.33 \%$ \\
\hline
\end{tabular}

Table 3. Crop correctness and completeness accuracy measures from DCRF max F1-score and MLC stack.

We further analyzed the grassland class where our approach has the lowest correctness compared to MLC-stack. Fig. 7 depicts completeness computed from each grassland validation parcel. Parcel numbers 8, 43, 49, and 51 have higher accuracy in MLCstack compared to DCRF max F1-score. However, observations from ground referencing photos in Fig. 8 demonstrate that errors encountered by DCRF max F1-score in those parcels are true positives. Hence, inhomogeneous grassland maps from DCRF max F1-score technique reflect true changes on ground not anticipated in ground reference data, see Fig. 8. Remaining parcels have comparable completeness accuracy in both methods.

A map of selected crops as classified by both MLC and DCRF max F1-score is shown in Fig. 9. It can be seen from the maps that DCRF max F1-score technique produces homogenous parcels compared to MLC-stack. This emphasizes the contribution of temporal phenological information inherent in images and spatial context in crop classification. The final map generated from DCRF max F1-score classification is illustrated in Fig. 10.

\section{DISCUSSION}

This study developed a DCRF crop classification technique from a sequence of TerraSAR-X images. In any classification, feature selection reduces computation demands. We selected four important features according to RF for crop classification. Features from VV-polarization were found important in crop discrimination compared to $\mathrm{HH}$-polarization as also established in Bargiel

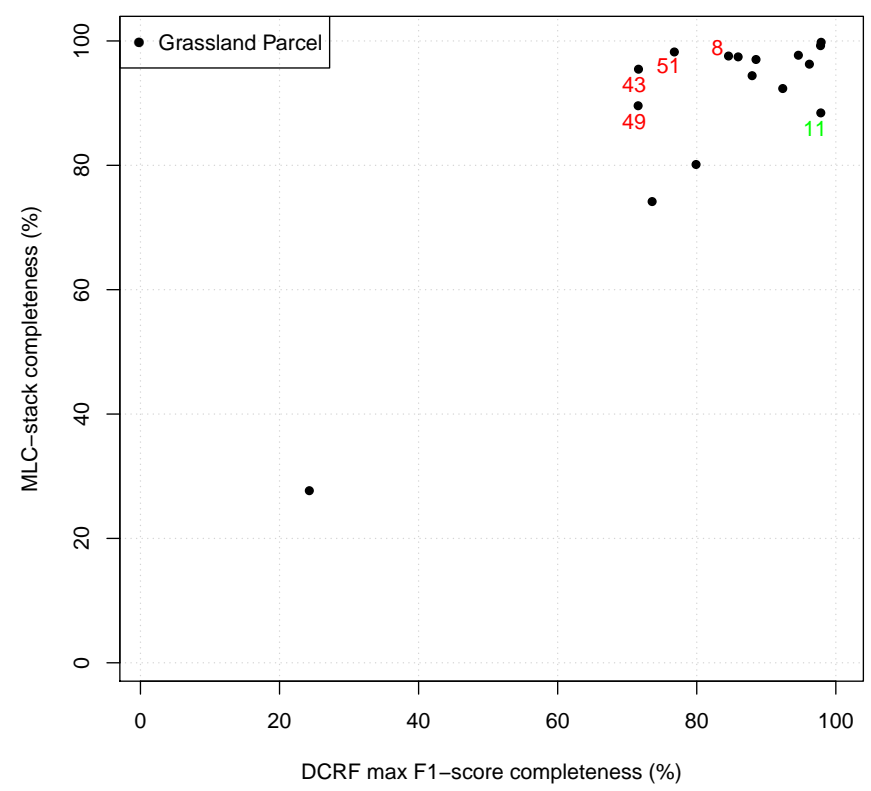

Figure 7. Scatterplot of DCRF max F1-score against MLC-stack completeness computed each grassland validation parcel.
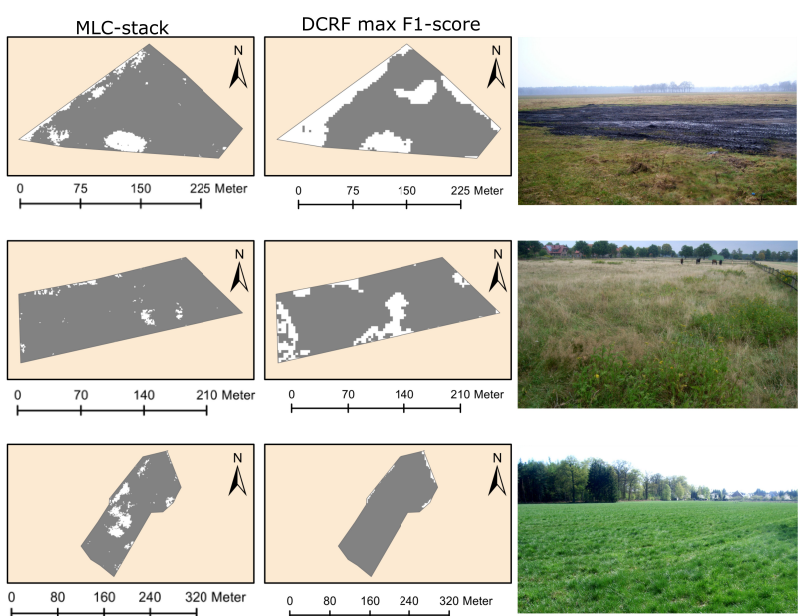

Figure 8. Grassland parcels as classified by MLC-stack and DCRF max F1-score and corresponding ground referencing photos. Top to bottom row corresponds to parcel numbers 49,8 , and 11 respectively as shown in Fig. 7. Grey and white areas correspond to grasslands and misclassifications respectively.

and Herrmann (2011). We exploited their synergy for crop classification. Our DCRF framework introduces spatial and temporal interactions. To enhance better data dependent spatial interactions we developed a new interaction term expanded from contrast sensitive model. Experimental results established that our new model is robust and suitable for crop classification. We set $\beta=10$ and $e t a=1$ based on a trade off between high classification accuracy and over-smoothing. Compared to contrast sensitive model proposed in (Shotton et al., 2009), the new model moderates smoothing given data evidence in two scenarios. First scenario, when adjacent labels are similar and data evidence support it, smoothness weight is increased. If data evidence does not support label similarity, smoothness weight is reduced proportional to difference in data. The second scenario considers dissimilar adjacent labels by reducing smoothness weight if supported by data evidence. When label dissimilarity is contrary to data evidence, smoothness weight is increased inversely proportional to the magnitude of data evidence. This design realized a 


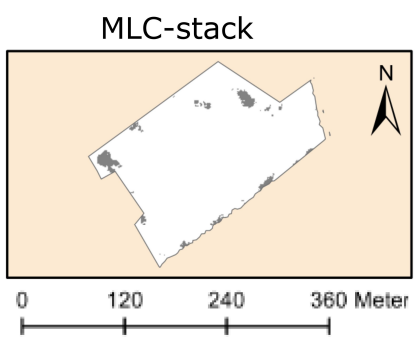

DCRF max F1-score
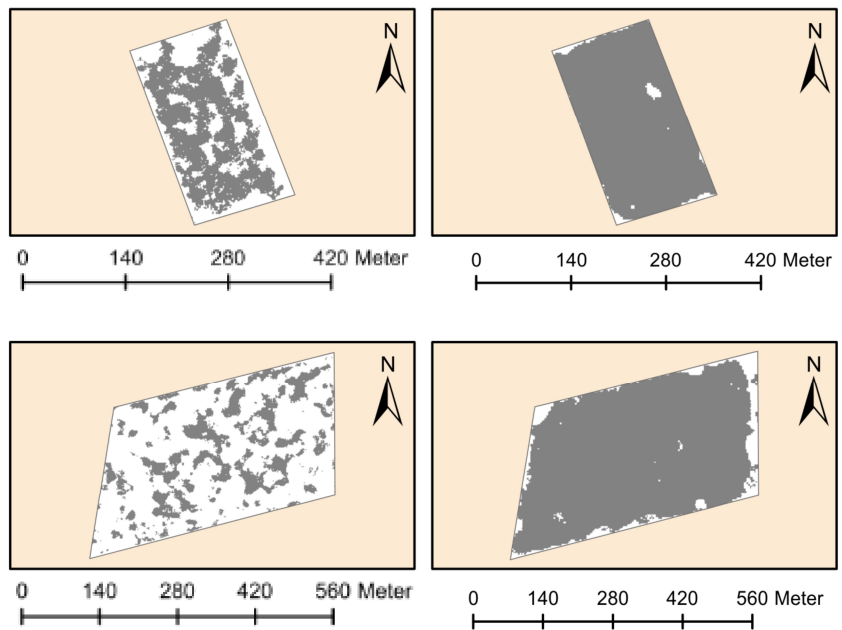

Figure 9. Maps of selected crops: top to bottom row represent oats, sugar beet, and maize parcels respectively. Correctly classified regions are illustrated in grey colour.

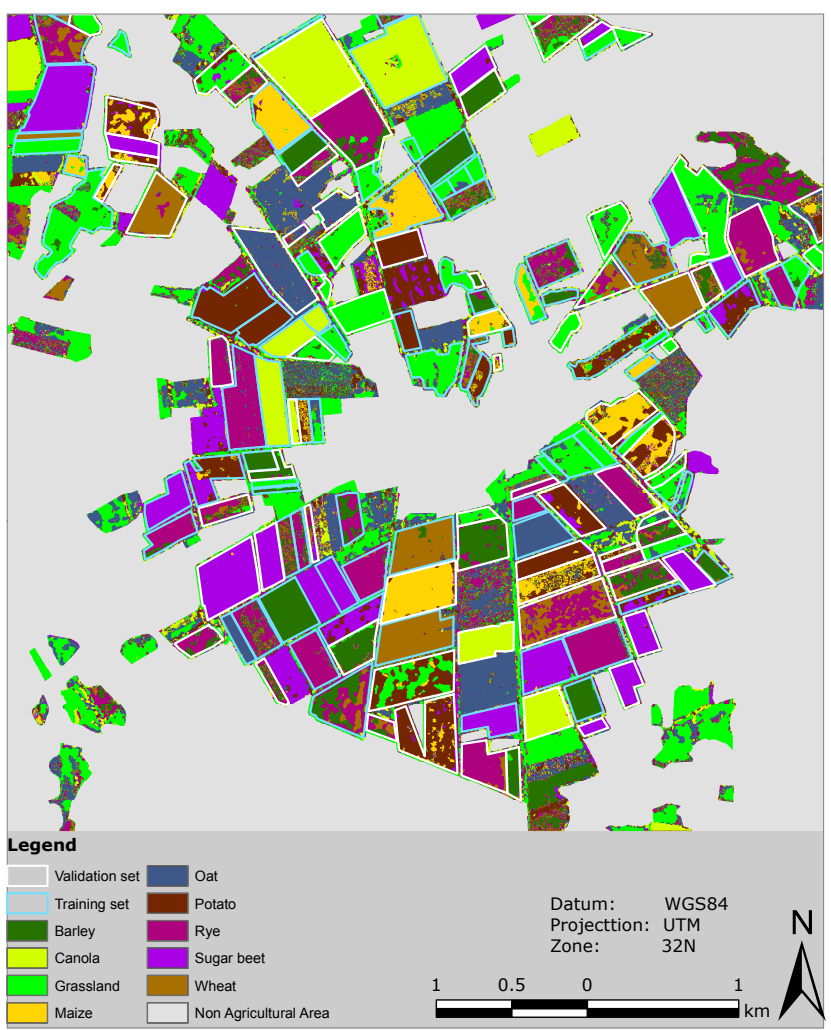

Figure 10. Crop map of Fuhrberg, Germany, from DCRF Max-F1 approach . Crop legend adopted from Ebinger (2012).

discontinuity adaptive model that moderates smoothing considering data evidence as suggested by $\mathrm{Li}$ (1995). Our model is differ- ent from contrast sensitive model which only favours smoothing similar adjacent labels.

The novel interaction potential model is adopted in CRF to design a DCRF sequence template classifier that considers inherent phenology in images. We established that including spatial and temporal phenological information improved classification accuracy in all epochs. Moreover, our technique still outperformed MLC and CRF classification methods utilizing merged multitemporal images as bands for classification (Table 2). To avoid a limited judgement using overall accuracy, we further compared our approach, DCRF max F1-score, with MLC using stacked images. Analysis of correctness and completeness exposed a detailed distribution of how each crop is recognized. DCRF max F1-score poorly classified grassland parcels compared to MLCstack. However, errors encountered by the method correspond to true ground changes that were not detected by MLC-stack for two reasons. First, MLC-stack classification places all features in one feature space which leads to a large variance that dominates small variations in a class. Two, DCRF max F1-score considers data and label dependent spatial interactions. This is supported by the fact that in a homogenous parcel, e.g. parcel number 11 in Fig. 8, DCRF max F1-score completely recognizes the parcel with higher accuracy than MLC-stack method, see Fig. 7. In addition, artificial changes or natural changes, e.g. due to different variety of grassland and changes in farm management as depicted by parcel number 49 and 8 in Fig. 8 respectively, are detected in DCRF max F1-score. In contrast, MLC-stack is a pixel based approach that ignores context. Thus, classification results from it are accompanied by "salt and pepper" effect (Fig. 9). All other grassland parcels were classified comparably well in MLC-stack and DCRF max F1-score because they are managed in a common and unique way, and driven by economic preconditions.

The advantage of our ensemble classifier is that it can be used with any number of available images at any time of a season to get an estimate of crop coverage. Moreover, compared to other classifier combination strategies, the weighting we introduced to the ensemble improved classification. Therefore, it guarantees an optimal map in terms of accuracy. This is essentially beneficial to governments and related stakeholders in food security policy formulations and seeking alternative preventive measures. In addition, agricultural stock market and traders can anticipate good years while insurers can accurately compute premiums and determine compensations where necessary.

\section{CONCLUSION AND OUTLOOK}

Our aim was to show that spatial-temporal information from a sequence of images improves crop classification. This was achieved by designing a DCRF classifier that realized spatial context and temporal phenological information exchange between nodes in a temporal neighbourhood. Spatial interaction significantly enhanced spatial dependency minimizing "salt and pepper" effect witnessed in MLC. The introduced data interaction term enforces a discontinuity adaptive model that moderates smoothing given data evidence.

On the other hand, stakeholder are not interested in several maps generated at each epoch in the same area. For them, statistics from one optimal map may be more relevant. Therefore, we designed a new ensemble approach based on F1-Score selection criteria and correctness weighting. The weighting schedule proved useful during integration of different classifier results.

So far, we let the DCRF model to automatically learn phenological information from data. The consequence of phenology 
is that a crop appears different at different times in satellite images. However, in some instances like preparation and post harvesting the backscatter is not entirely from the crops. Our future study will introduce phenological information from other external sources.

\section{ACKNOWLEDGEMENTS}

The project is funded by the German Federal Ministry of Education and Research (Project 50EE1326) and images provided by DLR.

\section{REFERENCES}

Bargiel, D. and Herrmann, S., 2011. Multi-Temporal Land-Cover Classification of Agricultural Areas in Two European Regions with High Resolution Spotlight TerraSAR-X Data. Remote Sensing 3(5), pp. 859-877.

Blaschke, T. and Strobl, J., 2001. What's wrong with pixels? Some recent developments interfacing remote sensing and GIS. GeoBIT/GIS 6(1), pp. 12-17.

Breiman, L., 2001. Random forests. Machine Learning 45(1), pp. 5-32.

Buja, K. and Menza, C., 2013. Sampling Design Tool for ArcGIS - Instruction Manual. NOAA, Silver Spring, MD.

Deutscher Wetterdienst, 2012. "Mittelwerte der Temperatur und des Niederschlags bezogen auf den aktuellen Standort". http: //www.dwd.de. (2 Oct. 2015)

Ebinger, L., 2012. "133 map categories! How the US Department of Agriculture solved a complex cartographic design problem". http://www.sco.wisc.edu/news/133-map-categorieshow-the-us-department-of-agriculture-solved-acomplex-cartographic-design-problem.html. (2 Oct 2015).

Forkuor, G., Conrad, C., Thiel, M., Ullmann, T. and Zoungrana, E., 2014. Integration of Optical and Synthetic Aperture Radar Imagery for Improving Crop Mapping in Northwestern Benin, West Africa. Remote Sensing 6(7), pp. 6472-6499.

Fritz, T. and Eineder, M., 2009. TerraSAR-X Ground Segment Basic Product Specification Document. Technical report, German Aerospace Center.

Hastie, T., Tibshirani, R. and Friedman, J., 2011. The Elements of Statistical Learning: Data Mining, Inference, and Prediction. Springer Series in Statistics, 2nd edn, Springer.

Hoberg, T. and Müller, S., 2011. Multitemporal Crop Type Classification Using Conditional Random Fields and RapidEye Data. In: ISPRS Workshop, Hannover, Germany.

Kenduiywo, B., Bargiel, D. and Soergel, U., 2015. SpatialTemporal Conditional Random Fields Crop Classification from Terrasar-X Images. ISPRS Annals 1, pp. 79-86.

Kenduiywo, B., Tolpekin, V. and Stein, A., 2014. Detection of built-up area in optical and synthetic aperture radar images using conditional random fields. Journal of Applied Remote Sensing 8(1), pp. 083672-1 - 083672-18.

Kittler, J., Hatef, M., Duin, R. and Matas, J., 1998. On combining classifiers. IEEE T. Pattern Anal. 20(3), pp. 226-239.
Kumar, S., 2006. Discriminative random fields. Int. J. Comput. Vis. 68(2), pp. 179-201.

Lafferty, J. D., McCallum, A. and Pereira, F., 2001. Conditional random fields: probabilistic models for segmenting and labeling sequence data. In: 18th ICML, Morgan Kaufmann, San Francisco, CA, USA, pp. 282-289.

Leite, P., Feitosa, R., Formaggio, A., da Costa, G., Pakzad, K. and Sanches, I., 2011. Hidden Markov Models for crop recognition in remote sensing image sequences. Pattern Recognit. Lett. 32(1), pp. 19-26.

Li, S., 1995. On discontinuity-adaptive smoothness priors in computer vision. IEEE T. Pattern Anal. 17(6), pp. 576-586.

Murphy, K., Weiss, Y. and Jordan, M., 1999. Loopy Belief Propagation for Approximate Inference: An Empirical Study. In: 15th Conference on Uncertainty in Artificial Intelligence, Morgan Kaufmann Publishers Inc., San Francisco, CA, USA, pp. 467-475.

OpenCV, 2014. Random Trees. http://docs.opencv.org/ modules/ml/doc/ml.html. (20 Nov. 2014).

Ozdarici-Ok, A., Ok, A. and Schindler, K., 2015. Mapping of Agricultural Crops from Single High-Resolution Multispectral Images-Data-Driven Smoothing vs. Parcel-Based Smoothing. Remote Sensing 7(5), pp. 5611-5638

Roscher, R., Waske, B. and Förstner, W., 2010. Kernel Discriminative Random Fields for land cover classification. In: IAPR Workshop on Pattern Recognition in Remote Sensing, pp. 1-5.

Shotton, J., Winn, J., Rother, C. and Criminisi, A., 2009. TextonBoost for Image Understanding: Multi-Class Object Recognition and Segmentation by Jointly Modeling Texture, Layout, and Context. Int. J. Comput. Vis. 81(1), pp. 2-23.

Siachalou, S., Mallinis, G. and Tsakiri-Strati, M., 2015. A Hidden Markov Models Approach for Crop Classification: Linking Crop Phenology to Time Series of Multi-Sensor Remote Sensing Data. Remote Sensing 7(4), pp. 3633-3650.

Sokolova, M., Japkowicz, N. and Szpakowicz, S., 2006. Beyond Accuracy, F-Score and ROC: A Family of Discriminant Measures for Performance Evaluation. In: Advances in Artificial Intelligence, Lecture Notes in Computer Science, Vol. 4304, Springer-Verlag Berlin Heidelberg, pp. 1015-1021.

Sonobe, R., Tani, H., Wang, X., Kobayashi, N. and Shimamura, H., 2014. Discrimination of crop types with TerraSAR-Xderived information. Physics and Chemistry of the Earth, Parts $A / B / C$.

Sutton, C., McCallum, A. and Rohanimanesh, K., 2007. Dynamic conditional random fields: Factorized probabilistic models for labeling and segmenting sequence data. Journal of Machine Learning Research 8, pp. 693-723.

Swain, P. H., 1978. Bayesian Classification in a Time-Varying Environment. IEEE T. Syst. Man. Cyb. 8(12), pp. 879-883.

Tilman, D., Balzer, C., Hill, J. and Befort, B., 2011. Global food demand and the sustainable intensification of agriculture. Proceedings of the National Academy of Sciences 108(50), pp. 20260-20264.

World-Bank, 2011. "Food Price Watch". http://www.worldbank.org/foodcrisis/ food_price_watch_report_feb2011.html. (22 Sept. 2014) 